\title{
Comparaison de deux modèles de profil de tige et validation sur un échantillon indépendant. Application à l'épicéa commun dans le nord-est de la France
}

\author{
Laurent Saint-Andréa, Jean-Michel Leban ${ }^{\mathrm{a}^{*}}$, François Houllier ${ }^{\mathrm{b}}$, \\ Renaud Daquitaine ${ }^{a}$ \\ ${ }^{a}$ Équipe de recherches sur la qualité des bois, Inra, 54280 Champenoux, France. \\ ${ }^{\mathrm{b}}$ Programme modélisation des plantes, Cirad, Inra, campus international de Baillarguet, BP5035, \\ 34032 Montpellier cedex 1, France.
}

(Reçu le 25 août 1997 ; accepté le 30 septembre 1998)

\begin{abstract}
Comparison between two stem taper equations, and validation on an independent sample. Case study of Norway spruce (Picea abies Karst.) in north-eastern France. Two stem taper equations are fitted and compared on a sample of Norway spruces (Picea abies Karst.) for which detailed data are available. Thomas's simple and trigonometric model behaves well at the bottom of the tree but strongly underestimates diameter at the top of the tree. The variable-exponent taper equation performs better along the stem, but also for extreme trees (height : diameter ratio less than 60 or greater than 120). The variable-exponent taper equation is validated on a large independent data set made up of 2577 trees from the National Forest Inventory, as this equation is to be used within the framework of the assessment of timber quality at a regional scale. The predicted and observed distributions of tree taper at $2.6 \mathrm{~m}$ and at the bottom of the tree are quite similar. A tree-to-tree comparison shows no major bias, except for small trees that have a height: diameter ratio of less than 70 . (C) Inra/Elsevier, Paris.)
\end{abstract}

stem profile / taper equation / model / validation / Picea abies

Résumé - Deux modèles de profil de tige sont ajustés et comparés sur un échantillon de 24 épicéas communs (Picea abies Karst.) pour lesquels on dispose de mesures intensives. Le modèle simple et trigonométrique de Thomas [30] s'ajuste très facilement et donne de bons résultats pour l'empattement des arbres, mais sous-estime fortement les diamètres en haut de l'arbre. Le modèle à coefficient de forme variable se comporte très bien tout le long des arbres, quel que soit leur élancement (hauteur/diamètre). Le modèle à coefficient de forme variable est ensuite validé sur un échantillon indépendant, 2577 arbres provenant de l'Inventaire forestier national. L'objectif est en effet d'utiliser ces équations dans le cadre d'une estimation de la qualité des bois d'une ressource sur pied. La validation montre que les distributions du défilement estimé à la souche et à $2,60 \mathrm{~m}$ sont très proches des distributions observées. La comparaison arbre par arbre ne montre pas de biais majeur, hormis pour le défilement à la souche et pour des arbres de faible diamètre et très trapus. ( $($ Inra/Elsevier, Paris.)

profil de tige / défilement / modèle / validation / Picea abies

* Correspondance et tirés à part

leban@nancy.inra.fr 


\section{INTRODUCTION}

La forme et le défilement des arbres sont souvent confondus dans la littérature. Par exemple, Larson [16] utilise sans les distinguer l'un ou l'autre des deux termes. Le défilement traduit en fait la décroissance du diamètre par unité de longueur (le tronc étant supposé dérivable), tandis que la forme prend aussi en compte des aspects tels que la courbure du tronc. La forme peut faire l'objet de mesures subjectives. Par exemple, Otegbeye, Samarawira [24] indicent les arbres de 1 (arbres droits ou rectilignes) à 6 (arbres présentant cinq courbures ou plus). Pardé et Bouchon [25] présentent un indice de forme défini par le rapport du volume de l'arbre sur le volume du cylindre de diamètre et de hauteur égaux à ceux de l'arbre : cet indice global traduit en fait le défilement. Lorsque la tige est assimilée à un cône, le rapport hauteur totale/diamètre à $1,30 \mathrm{~m}(h / d)$ peut aussi être considéré comme une mesure globale du défilement. $\mathrm{Ce}$ rapport sert également d'indicateur de stabilité des peuplements pour le sylviculteur et donne au forestier une indication de la sylviculture passée (notamment la densité de plantation). Dans ce travail, nous considérons uniquement le défilement, ou la décroissance métrique, sans tenir compte de la courbure des arbres.

Pour décrire le profil d'une tige, il existe deux familles de modèles : les modèles intégrés ou équations de profil de tige qui donnent directement le diamètre du tronc en fonction de la hauteur à laquelle on se place, et les modèles d'accroissement qui estiment d'abord le profil vertical de chaque cerne annuel (sa surface ou sa largeur) et, par sommation, permettent ensuite d'obtenir le profil de la tige.

Les modèles intégrés sont de loin les plus fréquemment utilisés et leurs équations peuvent être classées en trois groupes : $(i)$ les fonctions simples de défilement qui peuvent être des équations hyperboliques [1], trigonométriques [30], ou sigmoïdes [2, 5] ; (ii) les fonctions segmentées qui utilisent un ensemble de polynômes $[3,20]$; (iii) et les fonctions à exposant variable qui estiment le profil de tige à l'aide d'un coefficient de forme qui varie le long de l'arbre $[6,10,15,23]$. La plupart du temps, ces équations ont pour principal objectif l'estimation du volume des arbres et de la longueur des billons commerciaux, c'est-à-dire la part de l'arbre destinée au bois d'œuvre et au bois d'industrie. Ces équations sont souvent comparées les unes aux autres, en vue de tester leur aptitude à décrire le profil de tige de telle ou telle essence [23]. Elles ont aussi été utilisées pour modéliser le profil et le volume d'aubier du Douglas [19] ou pour prédire l'empilement des cernes à l'intérieur du tronc. Par exemple, l'équation de Houllier [12] sert dans un logiciel d'évaluation de la qualité des bois d'une ressource fores- tière existante (WinEpifn [28]) : l'empilement des cernes est obtenu en combinant un modèle moyen de croissance en hauteur et en grosseur avec la fonction de profil de tige ; c'est une reconstruction a posteriori de la croissance.

À l'inverse, le principe général des modèles d'accroissement est de simuler la croissance des arbres en reliant l'accroissement annuel du cerne le long de la tige à des caractéristiques fonctionnelles telles que le volume, la forme et l'extension du houppier [8, 11, 21, 27]. Par exemple, Mitchell [21] simule d'abord la masse foliaire des arbres compte tenu des voisins, il estime ensuite l'accroissement en surface des cernes. Deleuze [8] applique une démarche plus proche du fonctionnement et obtient une équation d'accroissement à partir d'un modèle de réaction-diffusion. Fourcaud et al. [11] ont proposé un modèle architectural couplé à un modèle de répartition des accroissements qui permet de simuler la croissance des arbres en hauteur et en diamètre. Cette dernière approche est actuellement utilisée comme support conceptuel pour analyser le processus de croissance des plantes.

Dans un contexte d'élaboration d'outils d'aide à la gestion, l'objet de cette étude est double : il s'agit de comparer deux modèles récents (tableau $I$ ) du type intégré $[6,30]$ en les ajustant sur l'épicéa commun dans le

Tableau I. Equations et paramètres des deux modèles.

$$
\begin{aligned}
& \text { Modèle } \\
& \text { Daquitaine } \\
& \quad d_{z}=d \cdot\left(\frac{h-z}{h-1.3}\right)^{q(h r, d, h)} \\
& \quad \text { Eù } h r=\frac{z}{h} \\
& \quad q(h r, d, h)=\theta_{1} \cdot(h, d)+\theta_{2} \cdot(h r-1)+\theta_{3} \cdot(h, d) \cdot e^{-\frac{\theta_{4} \cdot h r}{\theta_{3}(h, d)}} \\
& \theta_{1}(h, d)=\theta_{1,0}+\theta_{1,1} \cdot\left(\frac{h-1.3}{d}-70\right) \\
& \theta_{3}(h, d)=\theta_{3,0}+\theta_{3,1} \cdot\left(\frac{h-1.3}{d}-70\right)+\theta_{3,2} \cdot d
\end{aligned}
$$

Thomas

$$
\frac{d_{z}^{2}}{d^{2}}=b_{1}(h r-1)+b_{2} \sin (w \cdot h r \cdot \pi)+b_{3} \cot \left(h r \cdot \frac{\pi}{2}\right)
$$

où w est un coefficient propre à chaque espèce. 
nord-est de la France, puis de valider le modèle choisi sur un échantillon indépendant.

Le premier modèle est inspiré de Newnham [23] et son équation simplifiée est la suivante $: d_{h r}=u \cdot h r^{q(h r)}$, où $d_{h r}$ est le diamètre du tronc à une hauteur relative donnée $h r, u$ une constante et $q$ le coefficient local de forme. L'idée est de faire varier $q$ le long du tronc en fonction de la hauteur relative et des caractéristiques dendrométriques de l'arbre (hauteur totale et diamètre à $1,30 \mathrm{~m}$, par exemple). Ce type de modèle présente cependant trois désavantages : il est rarement intégrable analytiquement pour obtenir les volumes ; il est lourd à ajuster car non linéaire ; il a souvent du mal à décrire l'empattement des arbres $[6,23]$.

Le deuxième modèle a été propose par Thomas et Parresol [30] : il s'agit d'un modèle simple. Son équation : $d_{h r}=v \cdot f(h r)$, est une fonction linéaire en sinus et cotangente, où $v$ est une constante. Cette équation présente l'avantage d'être facile à ajuster et à intégrer. Par ailleurs, sa formulation trigonométrique est originale et le modèle semble capable de décrire correctement l'empattement des arbres, aussi bien pour Pinus elliottii que pour Quercus phellos.

Le second objectif de cette étude est de valider le modèle choisi dans une perspective d'évaluation de la qualité de la ressource en bois à une échelle régionale [13]. Il s'agit : (i) de vérifier aussi que le modèle est compatible avec les processus de croissance, c'est-à-dire que les profils de largeur (ou de surface) déduits de la succession de deux profils de tige (d'une année sur l'autre) sont satisfaisants ; (ii) d'étudier sur un grand échantillon représentatif de la ressource régionale le biais du modèle et ses éventuelles variations le long de la tige.

\section{MATÉRIELS}

Deux échantillons différents ont été utilisés. Le premier rassemblait 24 épicéas mesurés dans le cadre d'un projet européen [22]. Cet échantillon a servi à l'ajustement des modèles puis à une validation qualitative portant sur l'aptitude du modèle choisi à générer des surfaces d'accroissement réalistes. Le second provenait de l'Inventaire forestier national (IFN par la suite) et a servi à la validation quantitative du modèle.

\subsection{Notations}

Les notations suivantes sont utilisées :

- $a$ : âge de l'arbre,

- $h$ : hauteur totale de l'arbre,
- $d$ : diamètre à $1,30 \mathrm{~m}$,

- $c_{0.1}$ : circonférence à $10 \mathrm{~cm} \mathrm{du} \mathrm{sol,}$

- $d_{2.6}$ : diamètre de l'arbre à $2,60 \mathrm{~m} \mathrm{du}$ sol,

$-d_{\text {med }}$ : diamètre médian du billon compris entre $2,60 \mathrm{~m}$ et la découpe bois fort (mesure effectuée par l'IFN),

- Ih : longueur du houppier de l'arbre,

- $z$ : hauteur dans l'arbre, $0 \leq z \leq h$,

- $h r=z / h:$ hauteur relative,

- $d_{\mathrm{z}}$ : diamètre à une hauteur $\mathrm{z}$,

- $q$ : coefficient de forme local de la tige.

\section{2. Échantillonnage pour l'ajustement des modèles}

Les 24 arbres du projet européen [22] provenaient de deux peuplements expérimentaux qui différaient par leur indice de fertilité (fort ou faible) défini par la hauteur dominante à 50 ans. Dans chaque peuplement, douze arbres ont été prélevés en deux fois suivant le régime sylvicole : six dans une placette peu ou pas éclaircie et six dans une placette fortement éclaircie. Dans chaque placette, les six arbres ont été sélectionnés comme suit : deux arbres avec $l h \approx \overline{l h}+s$, deux arbres avec $l h \approx \overline{l h}$, deux arbres avec $l h \approx \overline{l h}-s$, où $\overline{l h}$ la moyenne des longueurs de houppier sur la placette, et $s$ l'écart-type de la population. Le tableau II donne les principales caractéristiques des arbres échantillonnés : tous étaient plutôt des dominants (houppier long) ou des codominants (houppiers courts), aucun n'était réellement dominé.

Pour cette étude du profil de tige, la circonférence a été mesurée tous les mètres environ, du bas de l'arbre au sommet. A des fins d'analyse de tige, une rondelle a aussi été prélevée tous les dixièmes de la hauteur de l'arbre.

\section{3. Échantillonnage pour la validation}

Pour valider les modèles, nous avons utilisé les données du deuxième inventaire réalisé par l'IFN en 1981 dans le département des Vosges. Ce département se situe au $2^{\mathrm{e}}$ rang français pour la ressource totale en Épicéa commun avec 16 millions de $\mathrm{m}^{3}$ et 58800 ha $[14,26]$.

Outre les mesures dendrométriques classiques (hauteur, âge, et diamètre à $1,30 \mathrm{~m}$ ), l'IFN mesure également le diamètre de la tige à $2,60 \mathrm{~m}$ de hauteur ainsi que la circonférence à la souche pour un certain nombre d'arbres. Au total, nous disposions de 2577 tiges dont 2449 pour lesquelles nous avions la mesure à la souche. Nous avons utilisé les tables de production de Décourt 
Tableau II. Caractéristiques des 24 arbres échantillonnés intensivement.

\begin{tabular}{|c|c|c|c|c|c|c|c|}
\hline Peupl. & $\begin{array}{l}\text { Fertilité } \\
\text { (indice) }\end{array}$ & $\begin{array}{l}\text { Intensité } \\
\text { des } \\
\text { éclaircies }\end{array}$ & Arbre & $\begin{array}{l}\text { Âge } \\
\text { (ans) }\end{array}$ & $\begin{array}{l}\text { Diamètre } \\
\text { à } 1,30 \mathrm{~m} \\
\quad(\mathrm{~cm})\end{array}$ & $\begin{array}{l}\text { Hauteur } \\
\text { totale } \\
\text { (m) }\end{array}$ & $\begin{array}{c}\text { Élancement } \\
\mathrm{h} / \mathrm{d}\end{array}$ \\
\hline 1 & Forte & Forte & 30 & 53 & 38,0 & 28,25 & 74,3 \\
\hline 1 & Forte & Forte & 39 & 53 & 31,5 & 26,67 & 84,7 \\
\hline 1 & Forte & Forte & 53 & 53 & 34,0 & 27,70 & 81,5 \\
\hline 2 & Forte & Faible & 72 & 53 & 31,0 & 29,13 & 94,0 \\
\hline 2 & Forte & Faible & 88 & 53 & 28,5 & 28,79 & 101,0 \\
\hline 2 & Forte & Faible & 94 & 53 & 30,0 & 29,10 & 97,0 \\
\hline 2 & Forte & Faible & 124 & 53 & 34,0 & 28,56 & 84,0 \\
\hline 2 & Forte & Faible & 170 & 53 & 26,5 & 28,20 & 106,4 \\
\hline 2 & Forte & Faible & 201 & 53 & 33,0 & 28,84 & 87,4 \\
\hline 3 & Faible & Forte & 459 & 63 & 43,5 & 27,80 & 63,9 \\
\hline 3 & Faible & Forte & 798 & 63 & 38,0 & 27,30 & 71,8 \\
\hline 4 & Faible & Faible & 128 & 63 & 33,0 & 23,70 & 71,8 \\
\hline 4 & Faible & Faible & 232 & 63 & 32,0 & 26,26 & 82,1 \\
\hline 4 & Faible & Faible & 472 & 63 & 34,5 & 27,07 & 78,5 \\
\hline 4 & Faible & Faible & 602 & 63 & 38,0 & 26,05 & 68,6 \\
\hline 4 & Faible & Faible & 654 & 63 & 31,5 & 25,93 & 82,3 \\
\hline 4 & Faible & Faible & 716 & 63 & 38,0 & 26,07 & 68,6 \\
\hline
\end{tabular}

[7] pour définir les classes de fertilité des peuplements en fonction de la hauteur dominante et de l'âge dominant. Il a ainsi été possible de ventiler les résultats en fonction des quatre classes de fertilité sélectionnées ( 1 à 4, dans la table de Décourt).

L'ensemble des simulations a porté sur ces 2577 arbres qui couvraient une large gamme d'âge, de hauteur, de diamètre à $1,30 \mathrm{~m}$ et de coefficient d'élancement ( $h / d$ ) (figures $l a$ à $l d$ ). Chaque classe de fertilité était également bien représentée (650 arbres en moyenne, figure le). On peut également noter un grand nombre d'arbres jeunes qui présentaient un faible diamètre : il s'agit des plantations effectuées dans le cadre des reboisements aidés par le Fonds Forestier National dans les années 1945 à 1960. Cette catégorie d'arbres n'était pas représentée dans le premier échantillon.

\section{MÉTHODES}

Dans la suite de cette étude, les deux modèles sont référencés par le nom de leur auteur : Daquitaine et Thomas (voir les équations dans le tableau I). Le modèle non linéaire (Daquitaine) a été ajusté par la procédure NLIN ([29]) et le modèle linéaire (Thomas) par la procédure REG du logiciel SAS. Dans le cas du modèle de Thomas, nous avons d'abord déterminé le coefficient $w$ propre à l'espèce par un ajustement non linéaire. Pour l'épicéa commun, le résultat de l'ajustement donne une valeur de 1,39 , qui est proche des valeurs obtenues par Thomas : entre 1,4 et 1,5 pour les résineux, deux pour les feuillus. Les autres coefficients du modèle ont ensuite été ajustés avec la procédure REG.

Pour comparer les modèles, nous avons étudié les résidus, c'est-à-dire la différence entre le diamètre mesuré $\left(d_{i, z}\right)$ et le diamètre estimé $\left(\hat{d}_{i, z}\right)$ à chaque niveau $z$ dans l'arbre $i$. Les quatre grandeurs suivantes ont été calculées pour chaque modèle : le biais,

$$
b=\frac{\Sigma\left(d_{i, z}-\hat{d}_{i, z}\right)}{n},
$$

l'erreur absolue moyenne,

$$
e=\frac{\Sigma\left|d_{i, z}-\hat{d}_{i, z}\right|}{n},
$$




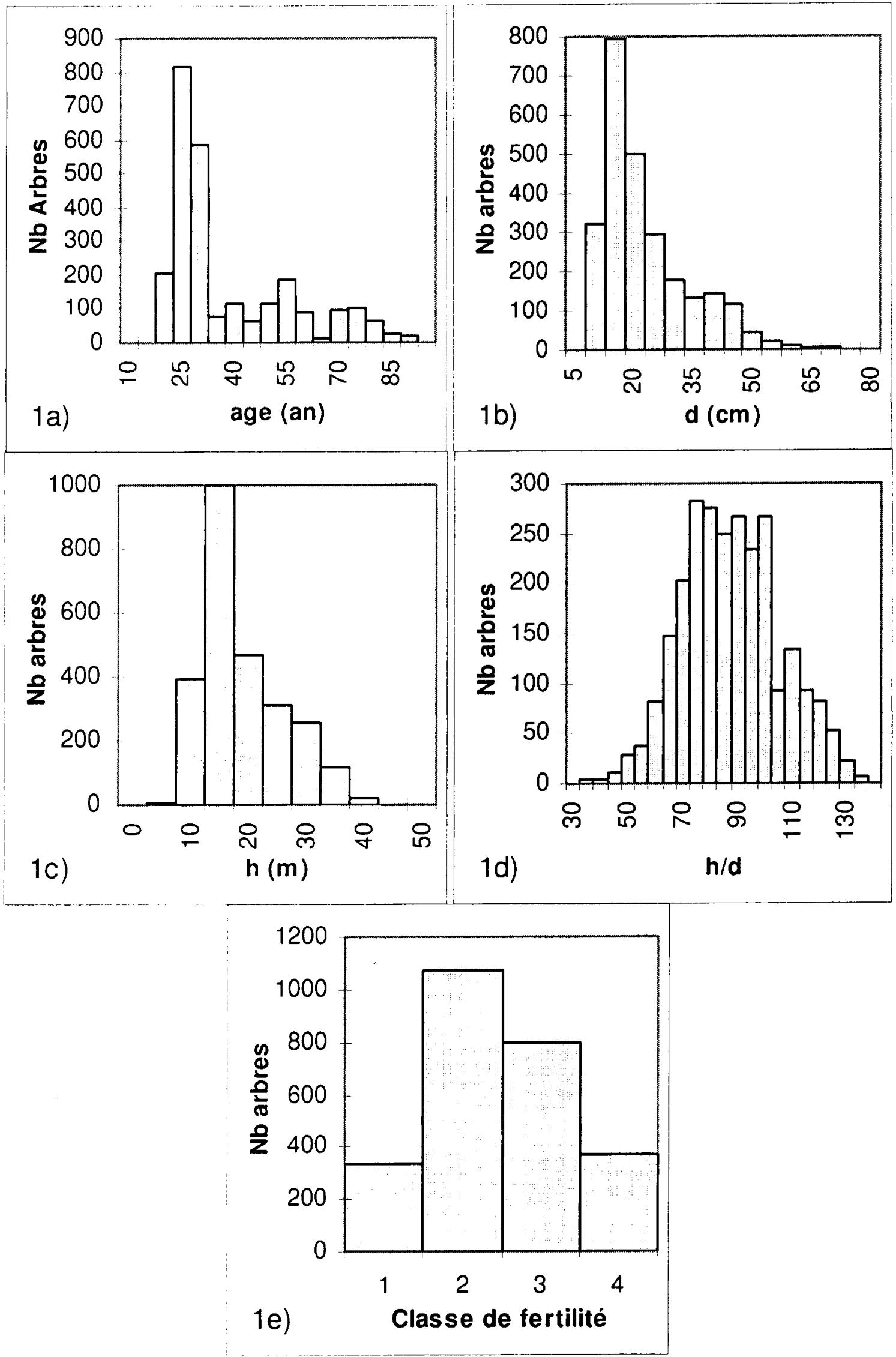

Figure 1. Histogrammes des principales variables de l'échantillon utilisé pour la validation (2 577 arbres). 
la racine de la moyenne du carré des erreurs,

$$
\operatorname{rmse} \sqrt{\frac{\Sigma\left(d_{i, z}-\hat{d}_{i, 2}\right)^{2}}{n}},
$$

et l'indice d'ajustement,

$$
R^{2}=\frac{\Sigma\left(d_{i, .}-\bar{d}\right)^{2}-\Sigma\left(d_{i, z}-\hat{d}_{i, z}\right)^{2}}{\Sigma\left(d_{i, z}-\bar{d}_{z}\right)^{2}},
$$

où $\bar{d}$, est la moyenne des diamètres mesurés et $n$ le nombre d'observations.

Afin de mettre en évidence les qualités et les défauts des modèles, ces valeurs ont également été calculées par classe de hauteur relative dans les arbres et par classe d'élancement $(h / d)$. Enfin, nous n'avons pas tenu compte, dans cette étude, de la structure particulière des erreurs (effet de l'arbre et autocorrélation le long de la tige).

\section{RÉSULTATS}

\subsection{Ajustements et choix d'un modèle}

Les paramètres des deux modèles sont tous significativement différents de zéro, sauf le paramètre $\theta_{3,2}$ du modèle Daquitaine. Il est probable que la faible variation des diamètres à $1,30 \mathrm{~m}$ des arbres utilisés pour ajuster le modèle en est la cause. Le modèle a donc été réajusté sans ce coefficient.

Le tableau III donne une synthèse des ajustements des deux modèles sur l'échantillon de 24 arbres. Pour l'ensemble, l'erreur absolue moyenne est proche du centimètre. Les quatre grandeurs définies précédemment montrent que l'équation à coefficient de forme variable (Daquitaine) s'ajuste mieux aux données que le modèle de Thomas. Globalement, ce dernier a tendance à surestimer les diamètres et il génère quelquefois des diamètres négatifs au sommet de l'arbre. Ce défaut, qui est signalé par Thomas \& Parresol [30], n'avait pas été corrigé dans leur étude puisqu'il est presque sans conséquence pour le calcul du volume de la tige. Cependant, dans le cas où cette équation de profil de tige est intégrée dans une succession de modèles comme c'est le cas dans le logiciel WinEpifn, ce défaut ne peut être ignoré, puisque les largeurs de cerne seraient aussi négatives.

La figure 2 montre l'évolution de l'erreur et du biais en fonction de la hauteur relative. Dans le bas de l'arbre, le modèle de Thomas se comporte bien hormis une légère surestimation des diamètres. En revanche, le modèle de Daquitaine génère des erreurs plus importantes dans le dixième inférieur de l'arbre. L'empattement est dans ce cas fortement sous-estimé. Pour tous les autres niveaux dans l'arbre, le modèle Daquitaine est moins biaisé et génère une erreur résiduelle plus faible que le modèle Thomas (figure $2 b$ ).

La figure 3 présente l'erreur et le biais moyen en fonction de l'élancement des arbres. Les deux modèles ont une erreur résiduelle et un biais plus importants pour les arbres à faible coefficient $h / d$, c'est-à-dire pour des arbres plutôt trapus. Le modèle Daquitaine est cependant plus souple que le modèle Thomas, notamment aux extrêmes $(h / d<70$ et $h / d>110)$. Cette propriété est importante lorsqu'il s'agit d'estimer la qualité des bois d'une ressource forestière existante $[17,18,28]$.

L'analyse des ajustement montre donc que le modèle Daquitaine est celui qui génère les erreurs les plus faibles, tant le long du tronc (hormis à la base de l'arbre) que pour une gamme étendue d'élancement des arbres. Le modèle de Thomas se comporte mieux au niveau de l'empattement mais a tendance à surestimer les diamètres sur une bonne partie du tronc et à sous estimer très fortement les diamètres à la cime de l'arbre (jusqu'à générer des diamètres négatifs). Par ailleurs, son mauvais comportement pour les arbres extrêmes (très coniques ou très cylindriques) en fait un modèle peu applicable pour estimer la qualité des bois d'une ressource forestière. En conséquence il n'a pas été retenu pour l'étape de validation.

Tableau III. Ajustement des deux modèles sur les 24 arbres échantillonnés intensivement.

\begin{tabular}{lccccc}
\hline Modèle & $\mathrm{dl}$ & $\mathrm{b}(\mathrm{cm})$ & $\mathrm{e}(\mathrm{cm})$ & $\mathrm{rmse}(\mathrm{cm})$ & $\mathrm{R}^{2}$ \\
\hline Daquitaine & 593 & 0,071 & 0,969 & 1,456 & 0,970 \\
Thomas & 596 & $-0,205$ & 1,359 & 2,030 & 0,942
\end{tabular}

Notations : $d l=$ degrés de liberté $; b=$ biais moyen ; $e=$ erreur absolue moyenne $; r m s e=$ Racine de la moyenne du carré des erreurs $; R^{2}=$ indice d'ajustement. 


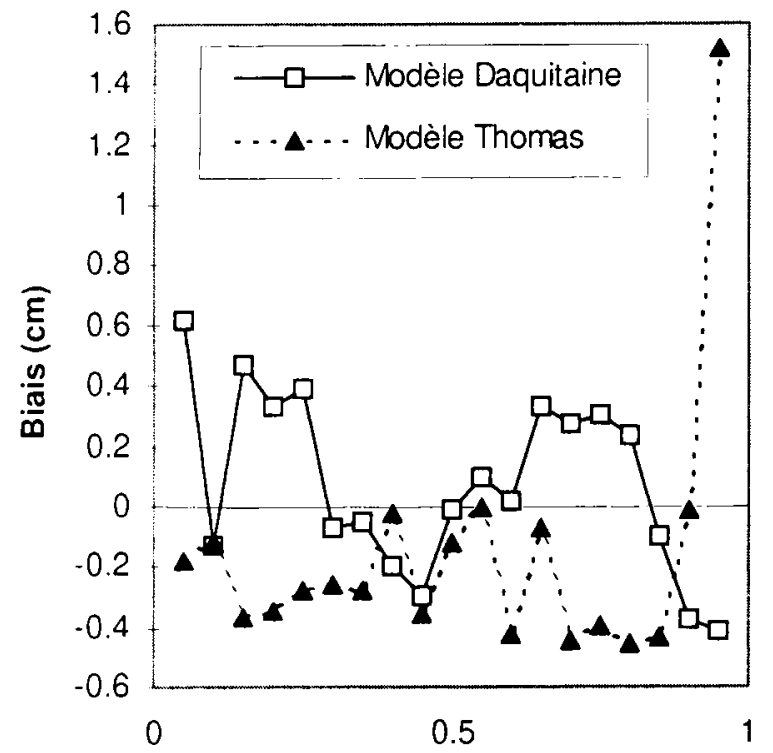

2a)

Hauteur relative

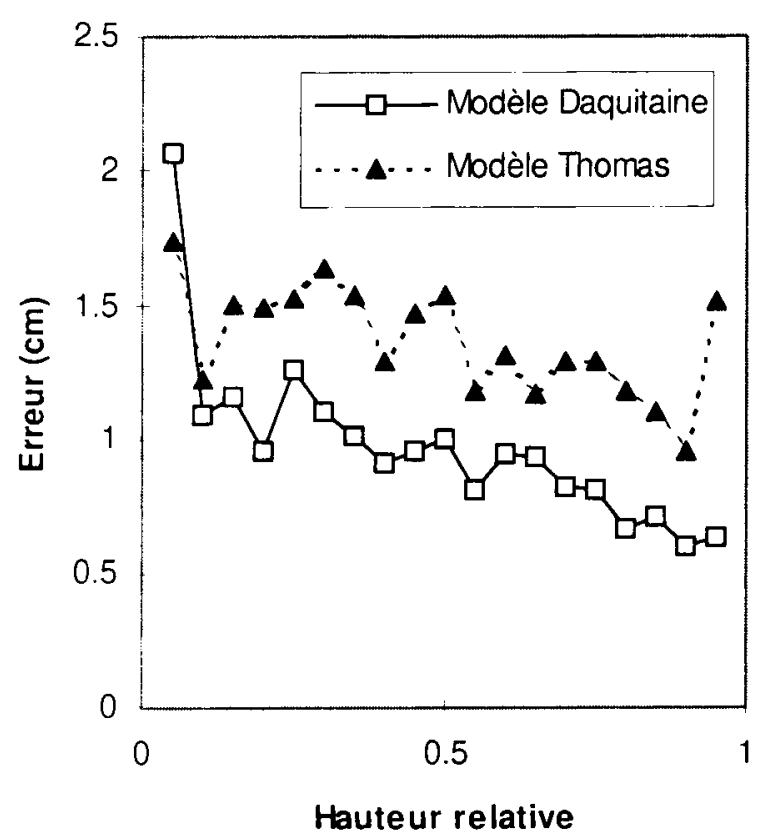

2b)

Figure 2. Biais et erreur absolue moyenne des deux modèles en fonction de la hauteur relative comptée depuis le sol ( $0=$ bas de l'arbre) sur l'échantillon de 24 arbres.

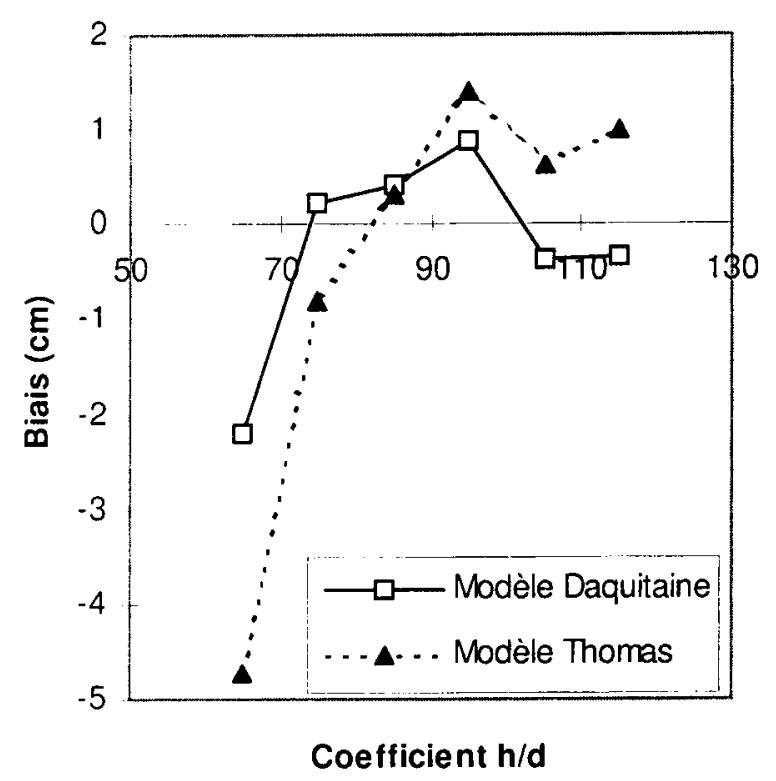

3a)

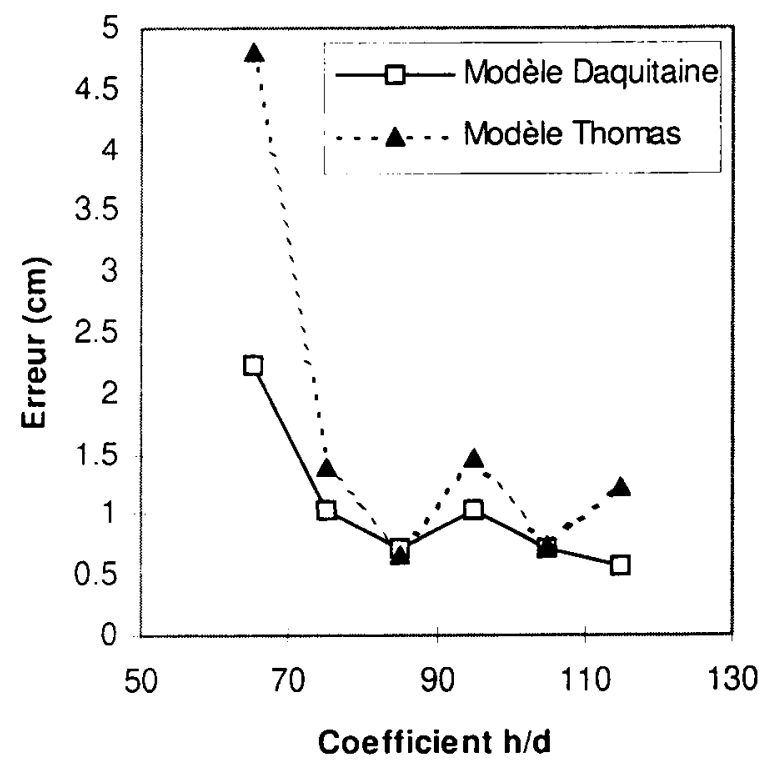

3b)

Figure 3. Biais et erreur absolue moyenne des deux modèles en fonction du coefficient $\mathrm{h} / \mathrm{d}$ des arbres sur l'échantillon de 24 arbres (toutes hauteurs confondues). 


\subsection{Estimation de la surface du dernier cerne par soustraction de deux profils de tige successifs}

Il n'a pas été possible d'utiliser les mesures de l'IFN pour cette première étape de validation puisqu'elles ne contiennent pas de mesures sur la structure interne des arbres. C'est donc le premier jeu de données qui a été utilisé. Pour chacun des 24 arbres, deux profils de tige successifs (à l'âge final et l'année précédente) ont été simulés pour étudier la variation de la surface du dernier cerne formé en fonction de la hauteur dans l'arbre.

La figure $4 a$ montre le résultat de ces simulations pour deux cas choisis aux bornes du faisceau des 24 courbes (arbres 472 et 209). Les résultats de ces simulations sont qualitativement bons au regard des mesures (figure $4 b$ ) : les largeurs de cerne sont toutes positives ; les ordres de grandeur sont satisfaisants ; la forme des profils simulés est bonne.

La figure $4 a$ montre aussi que la soustraction de deux profils de tige peut donner des profils de surface de cerne qui ne suivent pas la règle empirique dite « Loi de Pressler " [13]. En fait, cet écart est souvent observé dans la littérature : en général, les arbres en croissance libre présentent une surface de cerne qui augmente fortement dans le bas de l'arbre, tandis que les arbres dominés présentent des surfaces de cerne qui peuvent diminuer du haut vers le bas de l'arbre. Ces deux cas de figure sont rencontrés pour les arbres 209 et 472 qui présentent des coefficients d'élancement différents (55 et 79 respectivement). Cette aptitude à générer des profils non conformes à la loi de Pressler est donc favorable.

\subsection{Validation du modèle sur les données de l'IFN}

\subsubsection{Analyse globale des résultats}

Le tableau IV donne la moyenne, l'écart-type et le coefficient de variation du défilement prédit par le modèle Daquitaine, d'une part, et du défilement mesuré, d'autre part. Le défilement entre $1,30 \mathrm{~m}$ et $2,60 \mathrm{~m}$, $\operatorname{def}_{2,6}=\frac{d-d_{2,6}}{1,3}$, s'exprime en centimètres par mètre. Il en est de même pour le défilement à la souche : $\operatorname{def}_{0,1}=\frac{c_{0,1} / \pi-d}{1,2}$.

À la souche, les valeurs estimées sont nettement inférieures aux mesures. Cela confirme le résultat des ajustements où l'empattement des arbres était globalement sous estimé. Il faut préciser que le modèle a été établi sur des profils de tige dont les mesures près du sol n'étaient pas disponibles dans la plupart des cas (la première

\section{Surface des cernes en fonction de la hauteur relative. Simulations}

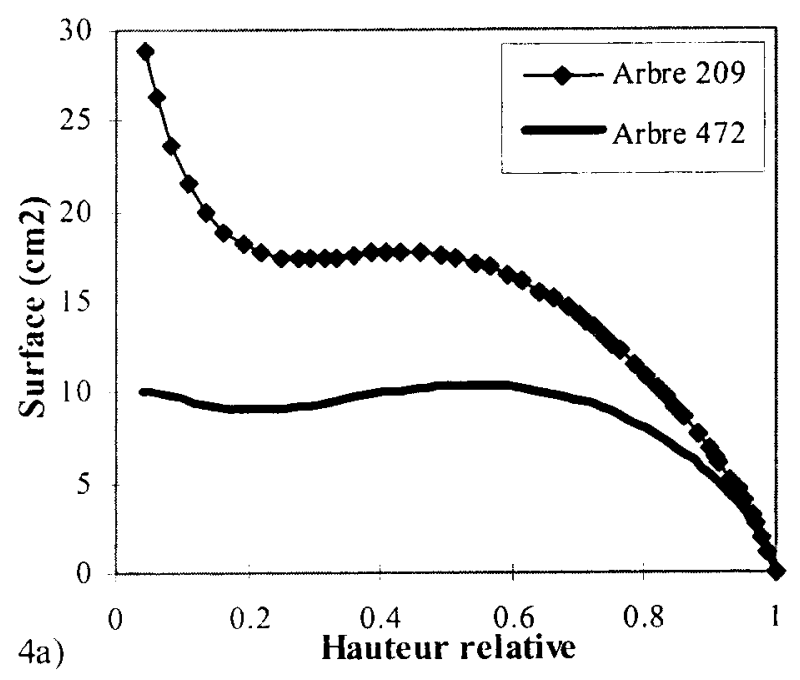

\section{Surface des cernes en fonction de la hauteur relative. Mesures}

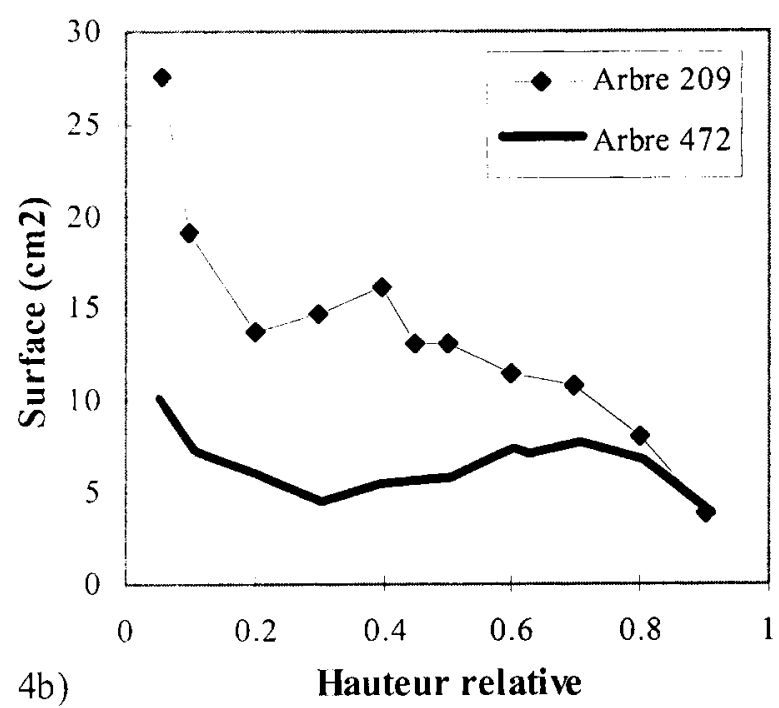

Figure 4. Profil de la surface du dernier cerne formé pour deux arbres du premier échantillon. 4a) Prédictions obtenues par soustraction de deux profils de tige successifs. 4b) Mesures obtenues par analyse de tige.

mesure étant effectuée à $50 \mathrm{~cm}$ du sol). Par ailleurs, il est également possible que la mesure de circonférence prise à $10 \mathrm{~cm}$ du sol surestime le diamètre moyen réel du fait des contreforts de l'arbre. 
Tableau IV. Analyse descriptive des défilements mesurés et prédits par le modèle Daquitaine sur les arbres IFN (2449 arbres pour $d e f_{1), 1}$ et 2577 arbres pour $d e f_{2,6}$ ).

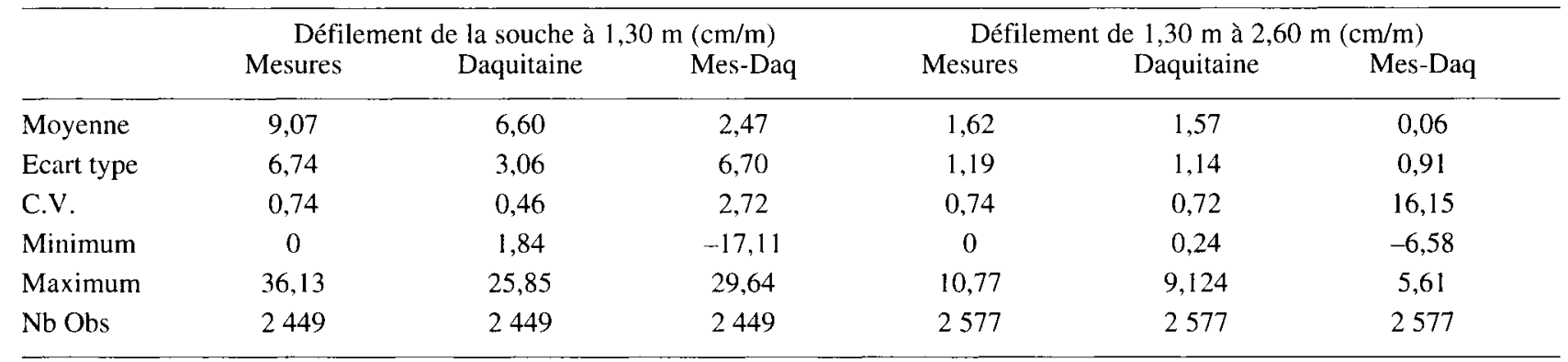

Tableau V. Ajustement du modèle Daquitaine sur les données de l'IFN et avec le terme $\theta_{3,2}: R^{2}=0,965, \mathrm{rmse}=2,6 \mathrm{~cm}$.

\begin{tabular}{lcccc}
\hline Paramètre & Estimateur & $\begin{array}{c}\text { Erreur } \\
\text { asymptotique }\end{array}$ & Limite inférieure & $\begin{array}{c}\text { Intervalle de confiance à 95 \% } \\
\text { Limite supérieure }\end{array}$ \\
\hline$\theta_{1,0}$ & 0,7525859 & 0,007235126 & 0,73840378 & 0,76676800 \\
$\theta_{1,1}$ & $-0,0040089$ & 0,000214986 & $-0,00443034$ & $-0,00358752$ \\
$\theta_{2}$ & 0,1541139 & 0,018771058 & 0,11731934 & 0,19090844 \\
$\theta_{3,0}$ & 1,6972525 & 0,092637264 & 1,51566725 & 1,87883766 \\
$\theta_{3,1}$ & 0,0770358 & 0,001618722 & 0,07386282 & 0,08020877 \\
$\theta_{3,2}$ & $\mathbf{2 2 , 0 1 3 3 0 3 0}$ & $\mathbf{0 , 2 1 0 0 2 1 7 4 2}$ & $\mathbf{2 1 , 6 0 1 6 2 3 7 7}$ & $\mathbf{2 2 , 4 2 4 9 8 2 3 2}$ \\
$\theta_{4}$ & 341,2329539 & 11,862737256 & 317,97991814 & 364,48598964 \\
\hline
\end{tabular}

En ce qui concerne le défilement à $2,60 \mathrm{~m}$, la gamme des valeurs prédites (minimum et maximum) correspond à celle mesurée. Il en est de même pour la moyenne et la variance des valeurs estimées qui se rapprochent beaucoup de celles calculées sur les mesures. Néanmoins l'écart-type du biais (Mes - Daq) montre qu'une part importante des variations des estimations n'est pas liée aux mesures (dans le cas idéal, le biais devrait être indépendant des estimations).

Une analyse par classe de fertilité montre que ces résultats sont identiques pour les peuplements fertiles et non fertiles aussi bien à la souche qu'à $2,60 \mathrm{~m}$. Il a été noté cependant qu'un certain nombre de défilements à 2,60 m était fortement surestimés pour les classes de fertilité $\mathrm{N}^{\circ} 4$. Ces valeurs étaient obtenues pour des arbres de faible diamètre $(<20 \mathrm{~cm})$ et de faible $h / d(<70)$, c'est-à-dire des arbres très coniques et pour la plupart assez jeunes $(<20-30$ ans).

\subsection{Ajustement du modèle sur les données de l'IFN}

Nous avons réajusté le modèle Daquitaine sur les données de l'IFN qui couvrent une vaste gamme d'âge et de dimension. L'objectif était notamment d'estimer le paramètre $\theta_{3,2}$ et de tester son signe. Pour chaque arbre, les diamètres à $10 \mathrm{~cm}$ de hauteur, $1,30 \mathrm{~m}, 2,60 \mathrm{~m}$ et au milieu de la tige étaient connus ainsi que la hauteur de l'arbre à la découpe $7 \mathrm{~cm}$.

Le tableau $V$ montre le résultat de cet ajustement. Le paramètre $\theta_{3,2}$ est significativement différent de zéro. Son signe positif permet de simuler des arbres plus nélö̈diques dans le bas de la tige. Malgré une précision moindre des mesures, l'écart-type résiduel reste faible (voir tableau $V$ ).

Les défilements des 2449 arbres de l'IFN ont été prédits ( $i$ ) avec le modèle ajusté sur les 24 arbres et (ii) avec le modèle ajusté sur les données IFN. Les valeurs pré- 
Tableau VI. Prédiction du défilement pour les 2449 arbres IFN à l'aide du modèle Daquitaine (i) ajusté sur les 24 arbres et (ii) ajusté sur les 2449 arbres.

\begin{tabular}{lccccccc}
\hline & \multirow{2}{*}{ Mesures } & \multicolumn{3}{c}{ Modèle ajusté sur les 24 arbres } & \multicolumn{3}{c}{ Modèle ajusté sur les données IFN } \\
& Simulations & Biais & rmse & Simulations & Biais & rmse \\
\hline def $_{0.1}$ & 9,066 & 6,599 & 2,467 & 7,14 & 9,123 & $-0,057$ & 4,04 \\
def $_{2,6}$ & 1,623 & 1,567 & 0,056 & 0,91 & 1,006 & 0,617 & 1,09 \\
def $_{\text {med }}$ & 0,826 & 0,898 & $-0,072$ & 0,20 & 1,035 & $-0,209$ & 0,28 \\
\hline
\end{tabular}

dites sont comparées aux mesures en termes de biais et d'écart-type résiduel (tableau VI). Les résultats du modèle ajusté à partir des données IFN sont globalement meilleurs : ils ne sont plus biaisés et sont plus précis au niveau de l'empattement ; ils sont beaucoup moins bons entre $1,30 \mathrm{~m}$ et $2,60 \mathrm{~m}$ (apparition d'un biais de $0,62 \mathrm{~cm} / \mathrm{m}$ sur le défilement et légère augmentation du rmse) ; la prédiction du défilement moyen entre $1,30 \mathrm{~m}$ et le diamètre médian est assez précise et peu biaisée (légère surestimation du défilement). Compte tenu de la faible précision des mesures effectuées par l'IFN (par comparaison aux mesures réalisées sur arbres abattus), ces résultats sont satisfaisants.

\section{DISCUSSION}

Le problème de l'empattement se retrouve quel que soit le type de modèle utilisé pour décrire le profil des tiges. Fonweban et Houllier [10] montrent, sur Eucalyptus saligna, que les équations de forme à coefficient variable ont le même défaut dans cette partie de l'arbre que les polynômes segmentés. Le tableau IV fournit le biais et l'erreur moyenne associés au modèle Daquitaine pour tous les arbres de l'échantillonnage IFN. Les résultats à $2,60 \mathrm{~m}$ sont excellents puisque l'erreur moyenne ne dépasse pas le centimètre. Cela confirme que sans estimation de points d'inflexion (à l'instar des polynômes segmentés), les modèles à coefficient de forme variable arrivent à bien décrire le bas de l'arbre. Les fortes sous-estimations à la souche proviennent de trois problèmes : $(i)$ des erreurs de calage en hauteur des mesures (le niveau de la souche est assez mal défini), (ii) le faible nombre de mesures entre la souche et $2,60 \mathrm{~m}$ (trois données) alors que c'est la zone où le défilement de la tige varie le plus, (iii) une gamme trop restreinte d'élancement des arbres (55 à 106 pour les 24 arbres du projet européen, contre 35 à 135 pour les arbres mesurés par l'IFN), (IV) une gamme trop restreinte de diamètre à $1,30 \mathrm{~m}$. Ce problème d'empattement a déjà été noté par Daquitaine [6] : il a montré que le modèle sans le terme $\theta_{3,2}$ surestimait l'empattement des petits arbres et sous-estimait l'empattement des gros (diamètre à $1,30 \mathrm{~m}$ compris entre 25 et $55 \mathrm{~cm}$ ).

Globalement, les résultats obtenus par le modèle Daquitaine sont satisfaisants. Dans le cadre de l'estimation de la qualité des bois d'une ressource sur pied, ce modèle possède la flexibilité souhaitée, c'est-à-dire une bonne aptitude à décrire le profil des troncs pour des arbres d'âge et de dimension variés et croissant dans des stations plus ou moins fertiles. On peut cependant émettre une réserve pour ce qui est de la modélisation de l'empattement.

Enfin, l'aptitude du modèle à générer des profils de surface de cerne corrects est importante puisque cela signifie qu'il peut être utilisé pour reconstruire a posteriori l'empilement des cernes dans le tronc. La notion de compatibilité a été introduite par Clutter [4] pour relier les modèles de production classiques (modèles intégrés du type tables de production) et les modèles d'accroissement (équations différentielles visant à décrire la réaction des peuplements à des traitements variés). La compatibilité signifie qu'un modèle intégré (ici, le profil de tige) peut être obtenu par sommation du modèle différencié (ici, le profil du cerne) et inversement. L'utilisation de modèles compatibles présente divers avantages. Le premier, et le plus évident, est la cohérence interne des modèles. Dans le cas où l'on dispose de bonnes connaissances ou de données solides pour l'un des points de vue (ici, le point de vue intégré avec le modèle de profil de tige), il est possible d'obtenir des informations sur l'autre point de vue (ici, le profil de largeur de cerne), voire de suggérer un modèle : dans le cas du modèle Daquitaine, la différenciation de l'équation par rapport au temps permet d'obtenir une équation de profil de cerne, malheureusement lourde, qui dépend de l'accroissement en hauteur et en diamètre de l'arbre. Enfin, on pourrait envisager d'aborder conjointement l'étude des profils de tige et des profils de cerne. 


\section{CONCLUSION}

Cette étude a permis de faire le point sur deux types de modèles de profil de tige. Le premier, simple et trigonométrique (Thomas), présente l'avantage de s'ajuster très facilement et donne de bons résultats pour l'empattement des arbres. En revanche, il a tendance à mal se comporter pour les niveaux supérieurs, génère quelquefois des diamètres négatifs au sommet de l'arbre et donne de fortes erreurs pour des arbres très coniques $(h / d<60)$ ou très cylindriques $(h / d>110)$. Par ailleurs, son interprétation en termes dendrométriques est difficile. En revanche, le modèle à coefficient de forme variable (Daquitaine) est plus facile à interpréter. L'exposant variable permet d'intégrer dans une seule équation les différentes géométries usuelles de tiges connues. Les termes qui la constituent sont reliés à la hauteur relative dans l'arbre et les paramètres dépendent des principales caractéristiques dendrométriques comme le diamètre à $1,30 \mathrm{~m}$ et le coefficient d'élancement. La validation quantitative sur un grand jeu de données montre une bonne estimation de la distribution des défilements. De plus la validation qualitative montre un bon comportement du modèle et semble satisfaire à la notion de compatibilité.

Cette étude a aussi permis de quantifier les erreurs générées par ces modèles en fonction de la hauteur relative dans l'arbre et en fonction de l'élancement des arbres. La connaissance de ces erreurs est importante car le modèle de profil de tige génère des diamètres qui sont ensuite utilisés pour prédire d'autres grandeurs (ex. largeur de cerne, densité du bois, etc.). Une étude de la propagation des erreurs permettra de quantifier l'impact des erreurs générées par le modèle de profil de tige sur les largeurs de cerne ou la densité du bois. Cette prochaine étude permettra de savoir si la précision et l'exactitude des modèles de profil de tige sont suffisantes pour qu'ils soient utilisés dans un enchaînement de modèles.

Enfin, nous n'avons pas tenu compte dans cette étude de la structure particulière des erreurs (effet arbre, autocorrélation le long de l'arbre). On peut envisager d'utiliser des modèles à effet mixte en tenant compte de l'autocorrélation des erreurs.

Remerciements: Cette étude a bénéficié du support financier de la région Lorraine et de la Communauté européenne au travers des contrats Forest MA2B-CT910024 et FAIR CT96-1915 (STUD). Nous remercions par ailleurs l'Inventaire forestier national et tout particulièrement Gérome Pignard pour ses conseils, ainsi que Jean Bouchon, Alain Franc, et Jean-Christophe Hervé pour leurs commentaires et suggestions.

\section{RÉFÉRENCES}

[1] Behre C.E., Is taper based on form quotient independant of species and size?, J. For. 22 (1924) 282-290.

[2] Biging G.S., Taper equations for second-growth mixed conifers of Northern California, For. Sci. 30 (1984) $1103-1117$

[3] Cao Q.V., Burkhart H.E., Max T.A., Evaluation of two methods for cubic-volume prediction of Loblolly Pine to any merchantable limit, For. Sci. 26 (1980) 71-80.

[4] Clutter J.L., Compatible growth and yield models for loblolly pine, For. Sci. 9 (1963) 354-371.

[5] Cormier K.L., Robin M.R., Czaplewski R.L., Bechtold W.A., Evaluation of weighted regression and sample size in developing a taper model for loblolly pine, For. Ecol. Manage. 53 (1992) 65-76.

[6] Daquitaine R., Création d'outils d'aide à la gestion pour des peuplements d'Epicéa commun en région Midi-Pyrénées. Rapport de stage de fin d'étude - Formation des Ingénieurs Forestiers, Engref, 1994, 69 pp.

[7] Décourt N., Tables de production pour les forêts françaises, Engref-Nancy, 1971.

[8] Deleuze C., Pour une dendrométrie fonctionnelle : Essai sur l'intégration de connaissances écophysiologiques dans les modèles de production ligneuse, thèse, $\mathrm{N}^{\circ} 090-96$, Université Claude Bernard-Lyon 1, 1996, 305 pp.

[9] Demaerschalk J.P., Kozak A., The whole-bole system: a conditioned dual equation system for precise prediction of tree profile, Can. J. For. Res. 7 (1977) 488-497.

[10] Fonweban J.N., Houllier F., Tarifs de cubage et fonctions de défilement pour Eucalyptus saligna au Cameroun, Ann. Sci. For. 54 (1997) 513-528.

[11] Fourcaud T., Blaise F., Barthélémy D., Houllier F., Reffye (de) P., A physiological approach for tree growth modelling in the software AMAPpara, in: Nepveu G. (éds.), Proceedings of the Second Workshop 'Connection between Silviculture and Wood Quality through Modelling Approaches and Simulation Softwares', Kruger National Park, South Africa, 26-31 August, Inra-Nancy, 1997, pp. 321-334.

[12] Houllier F., Silvicultural control and non-destructive assessment of timber quality in plantation grown Spruces and Douglas fir. Exploitation report of the Forest project contract $\mathrm{n}^{\circ}$ MA2B-CT91-0024. Modelling ring width in the tree in relation to silvicultural treatment. Final report of the task 2, Inra Champenoux, France, 1994,141 pp.

[13] Houllier F., Leban J.-M., Colin F., Linking growth modelling to timber quality assessment for Norway spruce, For. Ecol. Manage. 74 (1995) 91-102.

[14] Inventaire forestier national, Buts et méthodes de l'Inventaire forestier national, Ministère de l'agriculture, Paris, 1984,65 p.

[15] Kozak A., Smith J.H.C., Standards for evaluating taper estimating systems, For. Chron. 69 (1993) 438-444.

[16] Larson P.R., Stem form development of forest trees, For. Sci., Monogr. 5 (1963) 42 pp. 
[17] Leban J.-M., Modélisation de la croissance et de la qualité des bois. Estimation des propriétés des sciages d'une ressource forestière : Application à l'Épicéa commun (Picea abies Karst.), Rev. For. Franç. Numéro spécial XLVII (1995) 131-140.

[18] Leban J.-M., Daquitaine R., Saint-André L., Un outil d'évaluation de la qualité de la ressource en bois appliqué au Douglas : Le logiciel Win-Epifn, Forêt Entrep. 109 (1996) 11-15.

[19] Maguire D.A., Batista J.L.F., Sapwood taper models and implied sapwood volume and foliage profiles for coastal Douglas Fir, Can. J. For. Res. 26 (1996) 849-863.

[20] Max T.A., Burkhart H.E. Segmented polynomial regression applied to taper equations, For. Sci. 22 (1976) 283-289.

[21] Mitchell K.J., Dynamics and simulated yield of Douglas fir, For. Sci., Monogr. 17 (1975) 39 pp.

[22] Nepveu G., Silvicultural control and non-destructive assessment of timber quality in plantation grown Spruces and Douglas fir. Exploitation report of the Forest project contract $n^{\circ}$ MA2B-CT91-0024. Internal report of the Équipe de recherches sur la qualité des bois de I'Inra, Champenoux France, 1994, $26 \mathrm{pp}$.
[23] Newnham R.M., Variable-form taper functions for four Alberta tree species, Can. J. For. Res. 22 (1992) 210-223.

[24] Otegbeye G.O., Samarawira 1., Growth and form of Eucalyptus camaldulensis Dehnh. provenances in northern Nigeria, For. Ecol. Manage. 42 (1991) 219-228.

[25] Pardé J., Bouchon J., Dendrométrie. $2^{\mathfrak{e}}$ édition, Engref, Nancy, 1988

[26] Pignard G. Communication personnelle, 1998.

[27] Reffye (de) P., Houllier F., Blaise F., Fourcaud T., Essai sur les relations entre l'architecture d'un arbre et la grosseur des axes végétatifs. In : Ed. Inra Editions, Modélisation et simulation de l'architecture des végétaux. Bouchon J., Reffye de Ph., Barthélémy D., 1997, pp 255-424.

[28] Saint André L., Leban J.-M., Houllier F., Win EPIFN 1.0 : logiciel pour modéliser et simuler la qualité des bois d'une ressource forestière existante. Modèles pour l'épicéa commun. Pour PC sous Windows, Equipe de Recherches sur la Qualité des Bois, INRA 54280 Champenoux, France, Code APP, IDDN.FR.001.360012.00R.X.1996.000.00000, 1995.

[29] SAS Institue, Inc., SAS/STAT ${ }^{\circledR}$ User's Guide, Version 6, 4th edn., vol. 1, SAS Institute, Inc., Cary, NC, 1989, 943 p.

[30] Thomas C.E., Parresol B.R., Simple, flexible, trigonometric taper equations, Can. J. For. Res. 21 (1991) 1132-1137. 